proof-sheets of the questions received for this number will be sent to the correspondents so as to obtain their collaboration from the very beginning. Communications are to be addressed to the firm of Gauthier-Villars, 55, quai des Grands-Augustins, Paris; or to either of the editors: C.-A. Laisant, 162, avenue Victor-Hugo, and E Lemoine, 5, rue Littré, Paris. A. z.

Ayong the recent announcements of the Cambridge University Press we notice "'The scientific papers of John Couch Adams," vol. I., edited by Dr. W. G. Adams, F.R.S., with a memoir by J. W. L. Glaisher, and "A treatise on spherical astronomy" by Sir Robert S. Ball.

Dr. Charles H. Chapman has accepted the presidency of the University of Oregon, at Eugene, Oregon.

\title{
NEW PUBLICA'IIONS.
}

\section{HIGHER MATHEMATICS.}

Brewster (D.). Life of Sir Isaac Newton. New ed. (Castle Series.) London, Gall, 1893. 8vo. 340 pp.

28.

Carrara. Saggio d'introduzione alla teoria delle quantita complesse geometricumente rappresentate. Cremona, 1898

Chittienden (J. B.). A presentntion of the theory of Hermite's form of Lamés equation, with a determination of the explicit forms in terms of the $p$ function for the case $n=3$. [Diss.] Leipzig, (Teubner,) 1893.8 80. $85 \mathrm{pp}$. Mk. 2.80

Dewin (C.). Ueber zwei ebene Punktsysteme, die algebraisch auf einander bezogen sind. [Diss.] Lund, Moller, 1893. 8 vo. $85 \mathrm{pp}$. 1 plate.

Mk. 1.60

Fearrl. Ueber die unendlich oft iterierten Funktionen. [Progr.] Ostrau, 1898.

FrEGE (G.). Grundgesetze der Arithmetik, begrintsschriftlich abgeleitet. Vol. I. Jena, Pohle, 1893. 8vo. 32.and 253 pp.

Gravelius (H.). Lehrbuch der höheren Analysis. Zum Gebrauche bei Vorlesungen an Universitäten und technischen Hochschulen. Vol. I: Lehrbuch der Differentialrechnung. Berlin, Dummler, 1898. 8vo. 8 and 324 pp.

Mk. 6.00

Grchnipzen (F.). Die Affinitat in der Ebene nebst ihren speciellen Fülen, der Ähnlichkeit, Congruenz und Flächengleich heit, in unalytischer Behandlung. [Progr.] Brixen, 1893. 8vo. $27 \mathrm{pp.}$ Illustrated.

Huth (M.). Ueber Kurven von konstanter Steigung auf gegebenen Flitchen. [Progr. No. 578.] Stollberg, 1893. 410. 20 pp. 
JaHREsberich'T der Deutschen Mathematiker-Vereinigung. Heruusgegeben im Auftrage des Vorstandes von G. Cantor, W. Dyck, E. Lampe. Vol. II: 1892-3. Berlin, Reimer, 1893. 8vo. 4 and 156 pp. 2 plates.

Mk. 4.50

Obenrauch (F. J.). Monge, der Begrunder der darstellenden Geo. metrie als Wissenschaft. Eine mathematisch-historische Studie. [Progr.] Briunn, 1893. 8vo. 33 pp. 2.00

Prano (G.). Lezioni di analisi infinitesimale. 2 vols. Torino, Bocca, 1893. 8vo. $652 \mathrm{pp}$. Fr. 15.00

Preat y Lluch (P.). Problemas gráficos de estereometria. Madrid 1893. 8vo. 89 pp. Mk. 350

Thompson (H. D.). Hyperelliptische Schnittsysteme und Zusammen ordnung der algebraischen und transcendentalen Thetacharacteris. tiken. [Diss.] Göttingen, 1895. 4to. 33 pp. Illustrated.

Verzerchnis der seit 1850 an den deutschen Universităten erschienenen Doctor-Dissertationen und Habilitationsschriften nus der reinen und angewandten Mathematik. Herausgegeben auf Grund des für die Universităts-Ausstellung in Chicago erschienenen Verzeichnisses. Múchen, Dyck, 1892.

Mk. 1.70

\section{ELEMENTARY MATHEMA'IICS.}

Adam (W.). Geometrische Analysis und Synthesis. Eine Sammlung von 636 planimetrischen Konstruktions-Aufgaben mit reingeometrischer Lösung. Für höhere Lehranstalten, sowie zum Gebrauch beim Selbstunterricht systematisch geordnet und bearbeitet. $2 \mathrm{~d}$ ed. Potsdam, Stein, 1893. 8vo. 4 and $292 \mathrm{pp}$.

Mk. 4.00

BARDey (E.). Algebraische Gleichuugen nebst den Resultaten und den Methoden zu ihrer Auflösung. 4th ed. Leipzig, Teubner, 1893 8vo. 12 and $378 \mathrm{pp}$.

Mk. 6.00

Barthels (E. L.). Lehrbuch der Stereometrie und Trigonometrie in ausfuhrlicher Darstellung. Nebst einem Anhange, euthaltend die Regeln uber Potenz-, Wurzel-, Gleichungs-, Reihen- und Logarithmenlehre. Wiesbaden, Sadowsky, 1893. 8vo. 8 and $120 \mathrm{pp}$. Illustrated.

Mk. 2.80

BrigGs (W.) and EDmonson (J. W.). The geometrical properties of the sphere. (Univ. Corr. Coll. Tutorial Series.) London, Clive, 1893. 8 vo.

1s. $6 \mathrm{~d}$.

Briot (C.). Leçous d'algèbre. 16me éd., rev. par E. Lacour. Part II. Paris, 1893. 8vo.

Egamers (W.). Lehrbuch der darstellenden Geometrie. Part I: Die Elemente der darstellenden Geometrie. Leipzig, Seemann, 1893. 8vo. 8 and 52 pp. 5 plates. Boards.

Eмmenich (A.). Der Koordinatenbegriff und einige Grundlehren von den Kegelschnitten. Für den Schulunterricht bearbeitet. Essen, Baedeker, 1898. 8vo. 4 and 34 pp. 5 plates.

Genau (A.). Die Logarithmen und die ebene Trigonometrie. Büren, Hagen, 1893. 8vo. 47 pp. Illustrated. Boards. Mk. 1.10

HANDEL. Elementar-synthetische Kegelschittslehre. Berlin, Weidmann. 1893. 
Hadck. Lehrbuch der Stereometrie. Auf Grund vou Kommerells Lehrbuch neu bearbeitet. Fth ed. Tubingen, Laupp, 1898. 8vo. 226 pp.

Mk. 2.40

Hraler (J. F.). Methodisch geordnete Sammlung von Aufgaben aus der darstellenden Geometrie für Realschulen. Part III. Wien, Hölder, 1893 8vo. $49 \mathrm{pp}$.

Mk. 0.80

Hracher (B.). Lehrbuch der Geometrie zum Gebrnuche au Gymnasien, nach den neuen preussichen Lehrplänen bearbeitet. (3 parts.) Leipzig, Jacobsen, 1893. 8vo. 12 and $182 \mathrm{pp.}$ Mk. 8.00

Hertzer (H.). Funfstellige Logarithmentafeln. 3d ed. Berlin, Gaertner, 1893. 8vo. 4 and 88 pp. Boards. Mk. 1.20

Lackemann (B.). Die Elemente der Geometrie. Theil I: Planimetrie. 3d ed. Breslau, Hirt, 1898. 8 vo. $108 \mathrm{pp}$. Illustrated. Bonrds.

Mk. 1.25

Lengauer (J.) Die Grundlehren der ebenen Geometrie. Ein Leitfaden fur den Unterricht, mit Uebungsaufgaben. 4te Auflage der Geometrie von A. Stegmann. Kempten, Kösel, 1893. 8 vo. 8 and 180 pp. Illustrated.

Mk. 2.00

Matmhinsen (L.). Uebungsbuch fur den Unterricht in dèr Arithmetik und Algebra. Nach der Aufgubensammlung von Heis bearbeitet. $3 \mathrm{~d}$ ed. Köln, Du Mont-Schauberg, 1893. 8vo. 8 and $254 \mathrm{pp}$.

Mk. 2.00

Mrnk (W.). Lehrbuch der Geometrie als Leitfaden beim Unterrichte an höheren Lehranstalten. Theil I: Planimetrie. 9te Auflage, bear. beitet von E. Arndt. Berlin, Wiegandt und Schotte, 1893. 8vo. 6 and $110 \mathrm{pp}$. Illustrated.

Mk. 1.50

Mocnin (F. voN). Geometrische Formenlehre. 2d ed. Wien, Gerold, 1893. 8vo. 4 and 123 pp. Illustrated. Mk. 1.40

Jehrbuch der Geometrie. 3d ed. Wien, Gerold, 1893. 8vo. $178 \mathrm{pp}$. Illustrated. Cloth. Mk. 2.00

- Die geometrische Formenlehre in der Volksschule. Eine Anleitung für Lehrer zur Ertheilung des geometrischen Unterrichtes. th ed. Leipzig, Freytag, 1893. 8vo. $92 \mathrm{pp}$. Illustrated. Boards.

Nik. 1.10

RıBI (D.). Aufgaben über die Elemente der Algebra, methodisch georduet und in engem Anschluss an den Leitfaden von M. Zwicky. Herausgegeben von M. Zwicky. 3tes Heft. 6th ed. Bern, Schmid, 1893. 8vo. $32 \mathrm{pp}$.

Mk. 0. 50

RoEDer (H.). Lehrsätze und Aufgaben aus der Planimetrie. $2 d$ ed. Breslau, Hirt, 1893. 8vo. 96 pp. Boards. MIk. 1.00

Aufgaben aus der ebenen Trigonometrie. Aufösungen. Breslau, Hirt, 1893. 8vo. 62 pp.

Mk. 1.25

Rompele und Brins. Lehrbuch der Geometrie. Theil I: Planimetrie. 2d ed. Theil II: Trigonometrie und Stereometrie. Leipzig, Ahn, 1893.

Schlorke (J.). Lehrbuch der darstellenden Geometrie. 'Theil II: Schatten-und Beleuchtungslehre. Dresden, Kühtmann, 1893. 8vo. 4 and 60 pp. Illustrated.

Mk. 2.00

Schwering (K.). Anfangsgrinde der Arithmetik und Algebra für höhere Lehrunstalten, nach den neuen Lehrplänen benrbeitet. Frei. burg, Herder, 1893. 8vo. 8 and 80 pp. Cloth. 
Schwering (K.). Trigonometrie fur hohere Lehraustulten, wach den neuen Lehrplünen bearbeitet. Freiburg. Herder, 1893. 8vo. 8 and $52 \mathrm{pp}$. Illustrated. Cloth. Mk. 1.10

Streissler (J.). Die geometrische Formenlehre in Verbiudung mit der Anschnuungslehre. 8th ed. Triest, Schimpff, 1893. 8vo. 44 pp. 2 plates.

Mk. 1.20

Töpfer (H.). Iehrbuch der Planimetrie. Sondershausen, Eupel, 1898. 8vo. 4 and $188 \mathrm{pp}$.

Mk. 2.20

Verrens (K.). Abriss der darstellenden Geometrie. Theil I : Ortho gonalprojektivische Darstelluug von Punkten, Geraden, Ebenen und ebenflächigen Körpern. Chemnitz, Focke, 1893. 8vo. 4 and 84 pp. 15 plates.

Mk. 3.00

Wagner (H.). Lehrbuch der ebenen Geometrie und Aufgabensammlung für Realschulen. 2d ed. Hamburg, Grafe und Sillem, 1898. 8 vo. 8 and 176 pp. Illustrated. Boards.

Zwicky (M.). Grundriss der Planimetrie und Stereometrie nebst Uebungsaufgaben. Theil I: Planimetrie. Bern, Achmid, 1893. $8 \mathrm{ro}$ 5 and 88 pp. Illustrated. Boards.

Mk. 1.50

\section{APPLIED MA'THEMATICS.}

Chaperron (E.). Ueber die bewegende Kraft der Wärme. Deutsch herausgegeben von R. Mewes. Berlin, A. Friedlunder, 1893. 8vo. $48 \mathrm{pp}$.

Mk. 1.60

Crandall (C. L.). The transition curve, by offsets and deflection angles. London, 1893. 12mo. Mor.

Daltrop (V.). Die Gesetze des Fluges. Breslau, Max, 1893. 8vo. 44 pp. Illustrated.

Mk. 0.80

Dorr (R.). Pruktisch ausführbare Lösung des Problems der beliebigen Winkeltheilung. A. Beschreibung des Winkeltheilungsverfahrens, das ohne Anwendung eines besonderen Instruments auszufuhren ist. B. Beschreibung zweier fur dus deutsche Reich patentirter Winkeltheilungsinstrumente. Elbing, Meissner, 1893. 8vo. 14 pp. Illustrated.

Mk. 8.00

Enargser (F.). Die Zusatzkräfte und Nebenspanuungen eiserner Fachwerkbrücken. Eine systematische Darstellung der verschiedenen Arten, ihrer Grösse und ihres Einflusses auf die konstruktive Gestaltung der Brucken. Theil II : Die Nebenspannungen. Berlin, Springer, 1893. 8vo. 6 and 191 pp. Illustrated. Mk. 7.00

GaUss (F. G.). Polygonometrische Tafeln, zum Gebrauche in der Landmessung für die Teilung des Quadranten in 90 Grade zu 60 Minuten. Halle, Strien, 1893. 8vo. 194 and 28 pp. Cloth.

Mk. 12.00

Gotrschalk (A.). Conjugierte Poinsot-Bewegungon. [Diss.] Munster, 1893. 8vo. 29 pp.

Hoxamans (C.). Abhandlung nber die Unawive der Schwere. Deutsch von R. Mewes. Berlin, A. Friedlander, 1898 . 8vo. 10 and $47 \mathrm{pp}$. Illustraced.

Mk. 1.60

JaNuschkE (H.). Der Aetherdruck als einheitliche Naturkraft. [Progr.] Teschen, Prochaska, 1893. 8vo. 68 pp. Illustrated. Mk. 1.80 
KraK (W.). Vorträge uber Elasticitatslehre als Gruudlage für die Festigkeits.Berechnung der Bauwerke. (2 parts.) Hannover, Helwing, 1893. 8vo. 14 and $361 \mathrm{pp}$. Illustrated.

Mk. 10.00

Loner (S. L.). Solutions of the examples in the "Elements of Statics and Dynamics." London and New York, Macmillun, 1893. 16mo. 83: pp. Cloth.

$\$ 1.75$

MoAulay (A.). Utility of quaternions in physics. London and New York, Macmillan, 1893. 8vo. 14 and $108 \mathrm{pp.} \mathrm{Cloth.} \$ \mathbf{\$ 1 . 6 0}$

MaIss (E.). Zur Lehre von den krummlinigen Bewegungen eines materiellen Punktes. [Progr.] Wien, 1893. 8vo. 24 pp. Illustrated.

Neumann (C.). Die Huupt- und Brenn-Punkte eines Linsen-Systemes. Elementare Durstellung der durch Mobius, Gauss und Bessel begrundeten Theorie. 2d ed. Leipzig, Teubner, 1893. 8vo. 8 and $42 \mathrm{pp}$. Illustrated.

Mk. 1.20

Ort (K. vor). Vortrige über Baumechanik, gehalten an der $\mathbf{k}$. $\mathbf{k}$. deutschen technischen Hochschule in Prag. Theil III. Prag, Dominicus, 1893 . 8vo. 14 and 400 pp. Illustrated. Mk. 10.40

Paoloni (P.). Diseguo assonometrico. Milano, Hoepli, 1894. 16mo. $122 \mathrm{pp}$. Illustrated.

Poincané (H.). Vorlesungen uber Thermodynamik, redigirt von J. IBlondin. Deutsche Ausgabe von W. Jaeger und E. Gumlich. Berlin, Springer, 1893. 8 vo. 16 and 298 pp. Illustrated. Mk. 10.00

Rember (W.). Die Festigkeitslehre und ihre Anwendung auf den Maschinenbau, elementar behandelt. 2d ed. Herausgegeben von L. Hummel. Mitweida, Schulze, 1899. 8vo. 10 and 402 pp. 22 plates.

Mk. 10.50

Taylon (D. W.). Resistance of ships and screw-propulsion. With diagrams and tables. New York, Macmillan, 1893. 8vo. 234 pp. Cloth.

$\$ 3.70$

Trsserand (F.). Traité de mécanique céleste. Vol. III: Perturbations des plandtes d'après la méthode de Hansen; théorie de la lunc.. Paris, Gauthier-Villars, 1894.4 to.

Fr. 22.00

Watson (H. W.). A trentise on the kinetic theory of gases. $2 \mathrm{~d}$ ed. London, Frowde, 1893. 8 vo. $96 \mathrm{pp}$.

Webrer (W.). Werke, herausgegeben von der königl. Gesellschaft der Wissenschaften zu Göttingen. Vol. V: Wellenlehre; besorgt durch E. Riecke. Berlin, Springer, 1898. 8vo. 30 and 438 pp. 18 plites.

Mk. 18.00

Wenzen (L.). Ein Beitrag zur Schwingungstheorie elastischer Saiten. [Progr.] Klagenfurt, 1898 . $8 \nabla 0.30 \mathrm{pp}$. Illustrated.

Witrmane (W.). Statik der Hochbauconstructionen. Theil II: Holzund Eisenconstructionen. 2d ed. Munchen, Rieger, 1883. 8vo. 8 and 328 pp. 8 plates. Illustrated.

Mk. 12.00

Zrwer (A.). An elementary treatise on theoretical mechauics. Part I: Kinematics. New York, Macmillan, 1893. 8vo. 8 and 181 pp. Cloth. 